\title{
Ehrhart Polynomials of Matroid Polytopes and Polymatroids
}

\author{
Jesús A. De Loera • David C. Haws • \\ Matthias Köppe
}

Published online: 15 October 2008

(C) Springer Science+Business Media, LLC 2008

\section{Erratum to: Discrete Comput Geom DOI: $10.1007 / s 00454-008-9080-z$}

Due to a production error the article was published with mistakes in the paragraph following Theorem 1. The corrected paragraph appears below.

The computation of volumes is one of the most fundamental geometric operations and it has been investigated by several authors from the algorithmic point of view. Although there are a few cases for which the volume can be computed efficiently (e.g., for convex polytopes in fixed dimension), it has been proved that computing the volume of polytopes of varying dimension is \# $P$-hard $[9,17,26,30]$. Moreover it was proved that even approximating the volume is hard [19]. Clearly, computing Ehrhart polynomials is a harder problem still. To our knowledge there were only two previously known families of varying-dimension polytopes for which there is efficient computation of the volume. These two families are simplices or simple polytopes with a polynomial number of vertices (this follows from Lawrence's volume

The online version of the original article can be found under doi:10.1007/s00454-008-9080-z.

J.A. De Loera $(\bowtie) \cdot$ D.C. Haws

Department of Mathematics, University of California, Davis, CA 95616, USA

e-mail:deloera@math.ucdavis.edu

D.C. Haws

e-mail: haws@math.ucdavis.edu

M. Köppe

Department of Mathematics, Institute for Mathematical Optimization (IMO),

Otto-von-Guericke-Universität Magdeburg, Universitätsplatz 2, 39106 Magdeburg, Germany

e-mail: mkoeppe@imo.math.uni-magdeburg.de 
formula [30]). Note that for simplices, it is at least NP-hard to compute the whole list of coefficients of the Ehrhart polynomial, while recently [2] presented a polynomial time algorithm to compute any fixed number of the highest coefficients of the Ehrhart polynomial of a simplex of varying dimension. Theorem 1 provides another interesting family of varying-dimension polytopes whose volumes and Ehrhart polynomials can be computed efficiently. The proof of Theorem 1, presented in Sect. 2, relies on the geometry of tangent cones at vertices of our polytopes as well as a new, refined analysis of the evaluation of Todd polynomials in the context of the computational theory of rational generating functions developed by $[1-4,11,12,40,42]$. A nice introduction to these topics can be found at [5]. 